\title{
EICT Based Diagnostic Tool and Monitoring System for EMF Radiation to Sustain Environmental Safety
}

\author{
K Parandham \\ Aisect University, \\ Bhopal-Chiklod Road, \\ Raisen, Bhopal, India
}

\author{
RP Gupta \\ Aisect University \\ Bhopal-Chiklod Road \\ Raisen, Bhopal, India
}

\author{
Amitab Saxena \\ Aisect University \\ Bhopal-Chiklod Road \\ Raisen, Bhopal, India
}

\begin{abstract}
-the adverse effects of electromagnetic radiation from mobile phones and communication towers on health issues are being well documented today. However, exact correlation between radiation of communication towers and their radiation levels, are not monitored.

Aim of this paper is to study, analyze, apply networking and data mining technologies to develop an EICT based Diagnostic tool and Monitoring system for electromagnetic radiation levels into environment. This system is to network all mobile towers of each service provider as a single entity and then connect all service providers to a central monitoring agency online for continuous monitoring. Since very large numbers of mobile towers exist in India, each state can have its own regional network which is further networked with national central network. This can be enlarged to entire world for monitoring the EMF radiation levels near every mobile tower. For these regional national and international networks the connectivity is to be instituted by the respective service provider.
\end{abstract}

In this paper an attempt is made to logically apply Data Mining and networking technologies to develop a central EICT based diagnostic tool and monitoring system for EMF radiation from each transmission tower. With this system regional, national and international agencies/authorities can monitor the EMF radiation at each and every transmission tower area continuously and verify them with exposure standards. It is proposed to display this information using Integrated Display System in front of monitoring authority at appropriate levels.

Keywords-EICT Based Diagnostic tool; Electromagnetic Fields(EMF) Radiation; Mobile Telephony; Data Mining; Data Warehousing; Electronics; Information and Communication Technologies(EICT); International Commission on Non-Ionizing Radiation Protection(ICNIRP); Compressed Natural Gas (CNG)

\section{INTRODUCTION}

Exponential growth and developments in various fields of science and technology in the last few decades have intensified the human interface into the natural environment and associated physical, biological and ecological systems resulting in various unintended and undesirable impacts on environment, human health and society.

Electronics, Information and Communication Technologies (EICT) is ushering in a revolution in every field of daily life and the technological advantages brought to society are unimaginable. EICT is helping mankind in many ways and at the same time giving away many negative effects on environment, wildlife, human health and society at large. In addition, with the growth of mobile subscribers and their transmission towers, India is also witnessing a rapid population growth which is going to overtake China. For growing population the agricultural productivity and the problems influencing them should be of concern. The population of many species such as honey bees, which is one of the most important pollinator and useful factor for agricultural productivity, has seen a drastic population drop. In literature there is no much data about the effects of electromagnetic radiation available for most of our free living floral and faunal species in India.

Mobile communication industry is one of the fastest growing industries in the world. In recent years, there has been an exponential increase in the usage of mobile telecommunication devices, which has become an easy means for communication. The use of mobiles have become more conspicuous, during the last decade and this has led to construction of transmission towers in large numbers, built in urban, as well as in rural areas including other sparsely populated areas. Transmission towers are based on the electromagnetic waves, which over prolonged usage have adverse impacts on humans as well as on other fauna. The adverse effects of electromagnetic radiation from mobile phones and communication towers on health of human beings are being well documented today. Recently the electromagnetic fields from mobiles and other sources have been classified as "possibly carcinogenic to human "by the WHO,s International Agency for Research on Cancer (IARC). However, exact correlation between radiation of communication towers and wildlife, are not yet very well established.

\section{Historical BACKGROUND AND NeCESSITY}

The existing literature survey shows that the Electro Magnetic Radiations (EMRs) are interfering with biological systems in many ways. There had already been many warning bells sounded in the case of bees and birds, which probably heralds the seriousness of this problem, indicating vulnerability of other living beings as well. The electromagnetic radiations are being associated with the observed decline in the sparrow population in London and several other European cities (Balmori, 2002, Balmori, 2009, Balmori \& Hallberg, 2007) [1-3]. A vast majority of scientific literature published across the world serious effects of EMFs in various other species too. 
The pollution from EMRs being a relatively new environmental issue, there is a lack of established standard procedures and protocols to study and monitor the EMF impacts on humans especially among wildlife, which often make the comparative evaluations between studies difficult. In addition to the gap areas in research, the necessary regulatory policies and their implementation mechanism also have not kept pace with the growth of mobile communications industry. The present guidelines on exposure limits to EMF need to be refined since the ICNIRP Standard [4] currently followed in India is coined based on thermal effects of Radio Frequency and are dismissive of current epidemiological evidence on impacts of non-thermal nature on chronic exposure from multiple transmission towers.

Generally EMF Radiation hazards attributed are sleep disorders, headache, depression, discontent, irritability, nausea, dizziness, appetite loss, muscle spasms, numbness, tingling, altered reflexes, effects on eyes, brain, cells and many biological systems. Also birds loosing orientation and reduction in their population have been published after research in many countries. A new study from Brazil found the direct link to 4924 cancer deaths due to cellular antennas radiation between 1996 and 2006 which was published in 17 May 2011[5]. About $80 \%$ of the victims lived within $500 \mathrm{~m}$ away from cell phone towers. A study in ISREAL found that the incidence of cancer cases increased by four fold among people living within $350 \mathrm{~m}$ of transmission towers[6]. Another study in Germany found that a three fould increase in new malignancies among people living within 400m from a mast after 5 years of EMF exposures and 7 fold increase in cancer cases in Berlin[7-8]. Another study in Spain realized increased health problems among people living within $300 \mathrm{~m}$ from transmission towers[9]. Study by LB Despande in Sher-ePunjab colony in Andheri East, Mumbai, India found that many house wives have been diagnosed with many forms of cancer living within $91 \mathrm{~m}$ from a mobile tower. There are very large number of research papers and case studies are available on the negative impacts of Electromagnetic radiations on environment, human health, birds \& bees and wildlife. Most of them are quoted in expert group study report on the "Possible impacts of communication towers on wildlife including Birds and Bees", constituted by Ministry of Environment and Forests, Government of India[10].

Since the inception of mobile communication technologies, there have been concerns about the ill-effects of the mobile towers and mobile phones. Despite being a relatively newly acknowledged form of pollution, EMRs and their negative impacts on environment and biological systems have already been reported by several studies. However, most of the existing scientific literature on the negative environmental effects of electromagnetic fields, reports the results of experimental and epidemiological studies examining the serious impact on various aspects of human health. WHO and Government of India disapproves majority of these cases due to lack of proof. There is a conflict and difference of opinion exists among many agencies concerned about EMF radiation. One side Government agencies along with World Health Organization, another side cellular mobile operators along with manufacturers and third party is the society concerned with health issues. To resolve this issue initially monitoring of EMF radiation levels is the basic necessity so that mitigation activities can be initiated.

Therefore there is a strong case for design and development of EICT based diagnostic tool and on line monitoring system for EMF radiation in and around every transmission tower across the world. EICT can be effectively applied for design and development of diagnostic tool and monitoring system for continuous monitoring of EMF radiation from every transmission tower for ensuring environmental safety.

The large amount of varied EMF radiation data available across the country/world is to be surveyed, captured, meaningfully processed, sequenced, classified, structured, identified and hierarchically stored in various tiers based on policies decided. Data mining technology will be a solution involving high end advanced systems storage and state of the art software tools need to be included in the EICT Based Diagnostic tool and Monitoring system for continuous monitoring and analysis of EMF radiation levels from all the transmission towers. This information can be displayed in front of designated authorities through DLP for real time decisions to mitigate the irregularities in the EMF radiation patterns of any of the mobile service providers to confirm to safety standards. This requirement is that of the hardware, software and solution technologies.

\section{METHODOLOGY}

The data inputs considered for application to develop EICT based Diagnostic tool and Monitoring System for continuous monitoring of EMF radiation levels from each transmission tower is the radiated power density at specific distances.

The proposed methodology for networking of various EMF radiation values as inputs for EICT based Diagnostic tool and Monitoring system for on line monitoring EMF radiation levels around each transmission tower is an shown in figure.1, 2 and 3.

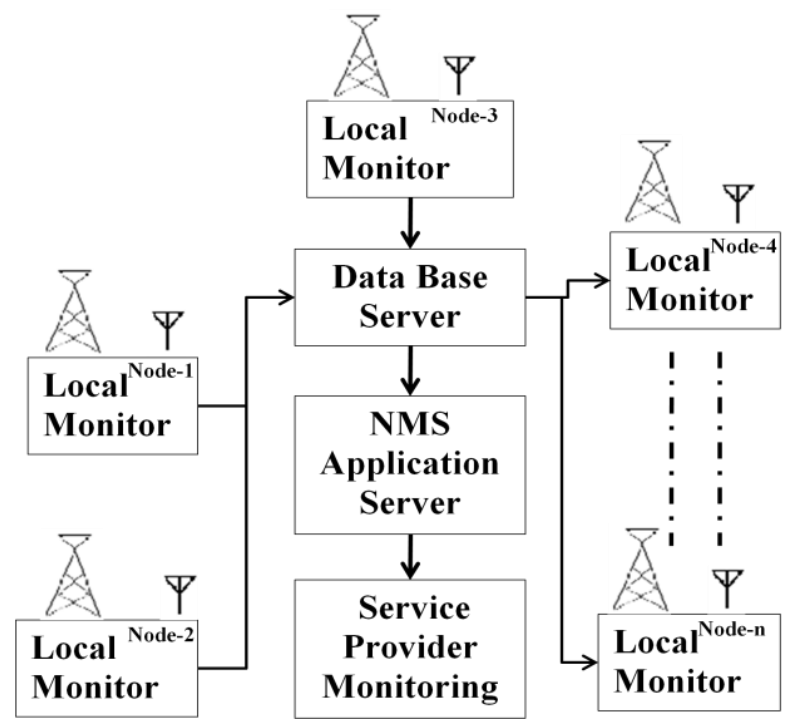

Fig. 1. Networking of EMF Radiation levels from each transmission tower service provider 


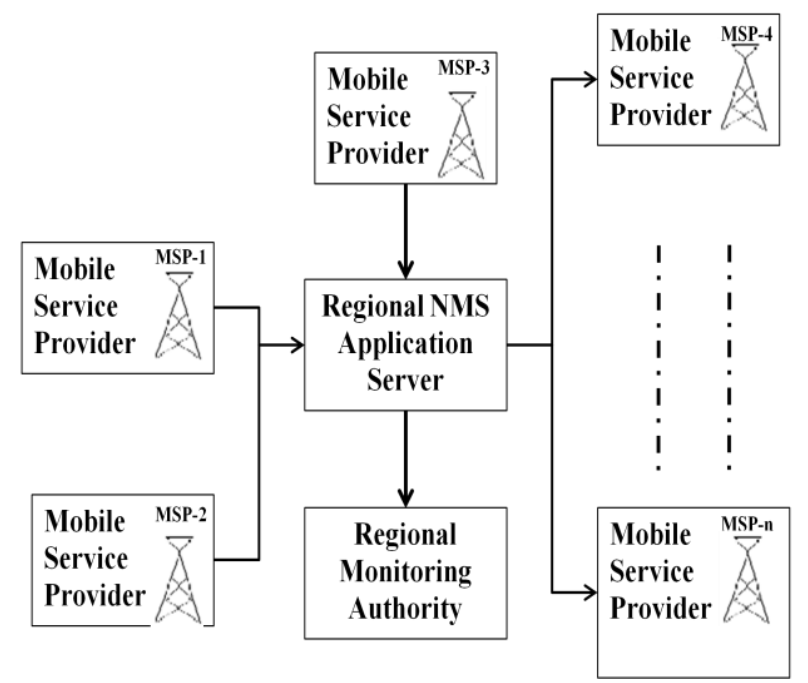

Fig. 2. Networking of EMF Radiation levels from each service provider in a region

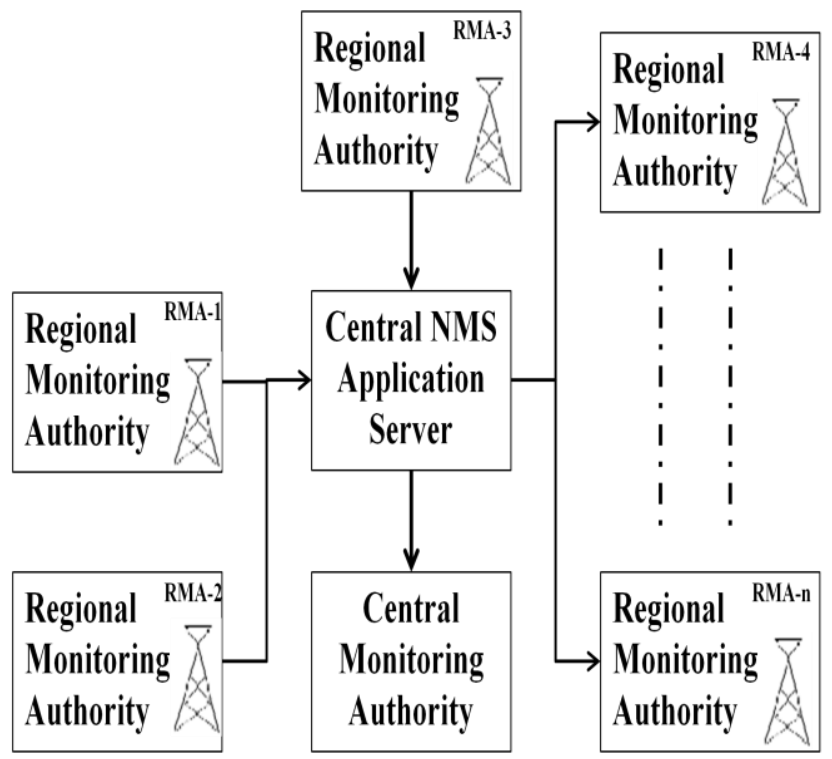

Fig. 3. Networking of EMF Radiation levels from each region to central monitoring authority

At every transmission tower wideband area monitor or smart monitor is envisaged at around $100 \mathrm{~m}$ distance. Frequency switching to monitor EMF radiation to be inbuilt into this system which can be selected from either locally, regionally or nationally through respective network management software. The local monitoring set up at each transmission tower can be termed as local monitoring node which is as shown in figure 1 .

From each monitoring node the measured EMF radiation data will be made to ride through respective service provider network. The EMF radiation data inputs from each node will pass through standard communication network such as multiplexer, sampling server, switch to database servers based at regional centre. The database servers are connected to NMS application server which is then connected through router to regional gateway server as shown in figure 1 .
Similarly all the cellular mobile service providers will provide respective EMF radiation inputs to regional gateway server of the regional monitoring authority. The monitored EMF radiation levels as inputs from each regional gateway server are linked to the central NMS application server. These data inputs then stored sequenced and displayed through a high end data wall display system.

These data inputs are required to be mapped in respect of each transmission tower, networked, processed, stored, and fused which are required to be displayed in front of monitoring authority. To integrate all these inputs for maximum situational awareness a multi-input, multi-window display processor is proposed in this paper. A variety of incoming EMF radiation levels will need to be displayed in a compact arrangement in front of monitoring authority for viewing for taking right decisions for mitigation of imbalance when compared to standard radiation limits accepted by the Government.

For this a display system that could combine multiple incoming inputs and display them using Digital Signal Processing, sound processing system, integrated controller, switcher, and Digital Light Processor based projectors, projecting the comparative EMF radiation levels and accepted standard limits on a glass beaded screen requirements are proposed in this research paper which are structured in figure 4.

\section{A. Salient Features of Customized Web NMS For This Application.}

The customized web Network Management Software (NMS) of this proposed EICT base Diagnostic tool and monitoring system for EMF radiations is to have the following salient features.

- Comprehensive monitoring capabilities to monitor EMF radiation levels, health of infrastructure, components, network protocols, system metrics with a single tool.

- Centralized view of entire status of EMF radiation levels selectively.

- Fast detector of alarming increase in the EMF radiation levels and their alerts to designated authorities.

- Alert acknowledgements provide communication to particular cellular mobile service provider on alarming EMF radiation levels and problem response.

- In case of regional NMS management facility to reduce / switch of EMF radiation power levels.

- Generation of historical reports and EMF radiation levels compared with standard radiation levels.

- Multiuser access to customized Web NMS interface to health department authorities, environmental specialists and department of commutation to view the EMF radiation levels.

- Extendable architecture for integration with in house and global applications. 
- Scales to monitor many regions and thousands of nodes.

- To have fail over capabilities to ensure nonstop monitoring.

\section{B. Data Mining Concepts Application.}

A high end advanced data capturing and analysis set up is proposed for this solution. The solution will involve high end advanced systems and storage and state of the art software tools for data analysis. The set of requirements proposed for this Diagnostic tool and monitoring system, EICT infrastructure needs are given below:-

- The EICT infrastructure should be capable of handling large data volumes of generally un-structured data available in various formats including but not limited to textual, video, audio and structured encrypted information.

- The total data volume will be in Gega Bites or in Tera Bites.

- The server infrastructure should be suitable for online analysis and processing and should be capable of handling large data volume providing near real time processing of large number of complex queries over the data volumes, generating required meaningful information. The input data flow will be from large numbers of transmission towers across the region and country from different mobile service providers.

- There should be provision for hierarchical storage, archiving, content management and data backup.

- The EICT infrastructure should be secure enough to the standards of security.

- The EICT infrastructure should provide support for data conversion from the available media to digital format and there should be appropriate mechanism for digital asset tracking.

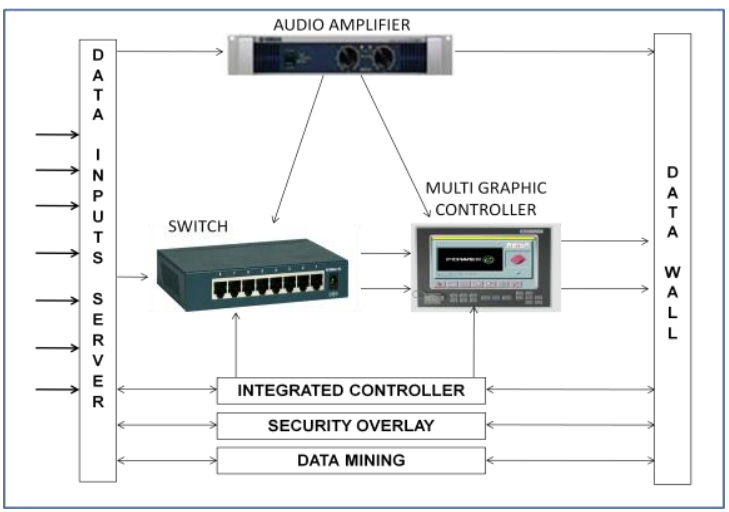

Fig. 4. Central Monitoring and Display System for EMF Radiation levels

- Data Inputs Server.This server receives and processes various EMF radiation inputs provided to the system and forward this information to various other subsystems.
- Switch. A network device used to interconnect various subsystems for intra-system communications and passage of various EMF data.

- Audio Amplifier. The amplifier is used to amplify the audio signal received by data input server and forward this multi graphic controller as well as over the network through switch.

- Multi Graphic Controller. This controller processes the multiple EMF graphic/video signals and multiplexes them with the amplified audio signal for further display over data wall.

- Data Mining. Analytical tool for analyzing data from different perspective and summarizing it into useful information after comparison with standard accepted levels. In addition data mining is also the process of finding correlations or pattern among dozens of fields in large relational database.

- Security Overlay. Architecture to ensure secure communications between the sub-systems/nodes and regions. It includes encryption, media secrecy as well as confidentiality.

- Integrated Controller. It provides enterprise protection for servers and enhances the server performance. It provides support for all RAID levels to ensure safety of critical EMF levels which have exceeded standard levels.

- Data Wall. Data wall is a big multi-screen LCD display system for graphics output as well as for audio-visual display. The data wall provides easy projection of critical information (EMF levels) for further assimilation and analysis.

\section{Data Fusion}

The data fusion solution will be comprising of the following three technologies:-

- Data Warehousing (DW) for capturing and organizing the EMF radiation data for fast near real time processing and retrieval of meaningful information.

- Content Management (CM) for classifying and managing EMF radiation levels in a well defined logical layout.

- Hierarchical Storage Management (HSM) for identifying, structuring and management of EMF radiation data from various cellular mobile service providers from different regions and nodes into various storage tiers based on the policies decided.

\section{RESUlTS \& DISCUSSION}

The IT infrastructure in figures 1, 2, 3 and 4 together forms the EICT based Diagnostic tool and Monitoring system for EMF radiation. The proposed system is to assist decision makers at regional and central (National) level to arrive at correct decision and have control over all cellular mobile providers at their disposal. This is an automated system for EMF radiation levels situation monitoring, to know status of 
all data inputs in that particular node, region and to arrive at right decisions at right time to mitigate higher or dangerous levels of EMF radiation.

The proposed network of networks is geographically distributed system. Distribution of proposed network based EMF radiation monitoring is key to management of impacts of this radiation which will have geographically distributed networks. This also makes network management efficient and scalable, enabling the central monitoring authorities to focus on the analysis of exceeding levels of radiation data collected across the nodes and regions of different cellular mobile service providers.

Distributed Mediation Servers (DMS) are servers that can be deployed along with central NMS server in large scale or remotely distributed network such as the one proposed in this paper. Each DMS server performs network facing functions for a regional monitoring network of each cellular mobile server provider. The collected EMF data can then be correlated to transfer relevant EMF information to the central NMS server.

In distributed scenarios distribution is effective in providing scalability and remote management capabilities to NMS management applications since cellular mobile services are expanding in an exponential growth.

When deploying NMS management application to manage a very large EMF radiation data monitoring networks, the application needs to be highly scalable to manage the nodal, regional devices, ports and connectivity, both logical and physical of different service providers. This scalability need can be addressed by the customized web NMS distributing the network facing the functionality via the distributed mediation servers. The central EMF radiation monitoring NMS server can collate EMF radiation levels from different distributed mediation servers of different regions and provide a single console view of the entire network to the monitoring authority.

When NMS management applications are deployed by cellular mobile service providers, they do require management of their IT infrastructure health monitoring along with EMF radiation levels across multiple/remote locations. Here only EMF radiations are required to be transferred to regional or central monitoring location which can be addressed in NMS application server by deploying the distributed mediation servers at remote locations.

In a distributed set up like in the proposed EICT based diagnostic tool and monitoring system for EMF radiations the following components are very significant while deploying customized web NMS using DMS servers.

- Central Server

- Distributed Mediated Server and

- User interface

The central server here is a logical server and is made up of two servers, namely the back-end server and the front-end server. Front-end servers are servers to which the monitoring authorities and the DMS are connected. The numbers of frontend servers depend on the planned performance and scalability metrics. The back-end servers store all the networks EMF radiation data in a centralized database and processes requests from monitoring authorities through the front-end servers. Both these servers are located at the central monitoring site. The collated EMF radiation data stored in a local database and correlated by the DMS. This ensures that only summary and critical data are sent to the central customized web NMS server. User interface facilitates the monitoring authority to connect to the DMS server at the specified node of particular region of the selected cellular mobile service provider to view the EMF radiation data available in the DMS database.

Network Management software for this proposed system is required in an operational environment where large amounts of information and sources need to be managed. The built in automation features of the software will be required to allow operators in the control room to focus fully on the important tasks. It will allow operators in the control room to focus fully on the important tasks. It has to allow right integration with custom applications, so that operators do not have to learn new tools or user interfaces after installation.

\section{CONCLUSION}

The world's entrance into the mobile telephony certainly had a profound impact on our society. It seems clear that the trend will continue to expand in many ways that will, no doubt, continue to surprise us. The expanding potential, however, is not an unmitigated blessing. It is very evident that there will be both positive and negative effects on society and environment.

The diagnostic tool and monitoring system proposed in this paper is an ideal solution for online monitoring of EMF radiations across all nodes, regions of different cellular mobile service providers to ensure environmental safety. This can give instant inputs of exceeding EMF radiation levels in any of the nodes of any region of any cellular mobile service provider. This can monitor in near real time and will be a great aid for knowing the EMF radiation levels across the nation.

However a diagnostic tool and monitoring system for respective controlling agencies such as Medical, Communication, Environment, Nongovernmental Organizations, Cellular mobile service providers and general public is felt appropriate for mitigating the higher EMF radiation levels.

A warehouse is more than an archive for data and more than a new way of accessing data. A warehouse is a subject oriented repository and provides tools to satisfy the information needs, not just for complex data queries, but as a general facility for getting quick, accurate, and often insightful information. It is to be designed in such a manner that users can recognize the information they want and access that information using this tool.

As per ICNIRP [11] guidelines EMF exposure limit in Canada is $3 \mathrm{w} / \mathrm{m}^{2}$, in India it is $9.2 \mathrm{w} / \mathrm{m}^{2}$ (now $0.92 \mathrm{w} / \mathrm{m}^{2}$ ), $2 \mathrm{w} / \mathrm{m}^{2}$ in Australia, $0.09 \mathrm{w} / \mathrm{m}^{2}$ in Germany and $0.001 \mathrm{w} / \mathrm{m}^{2}$ in Austria and in New South Wales(Australia) it is $0.00001 \mathrm{w} / \mathrm{m}^{2}$. Also successful communication with GSM mobile established in Germany [12] with 0.001 microwatts per square meter. A mobile phone requires -80 to $-100 \mathrm{dbm}$ power for its 
operation. Thus it is seen that at a distance of $50 \mathrm{~m}$ the power level is 50 to $60 \mathrm{dbm}$ higher in reality, meaning 1, 00,000 to $10,00,000$ times more power is radiated for mobiles operation. This is gravely hazardous to environment, health and to society.

Until now, society has been absorbing the harmful, invisible EM radiations without even being aware of it. With exponential growth in mobile communications this EM radiation pollution has started showing ill effects on environment, health of human beings, birds, bees and animals. Hence there is an urgent requirement to take precautionary steps to safe guard the environment and society. In this first step is to monitor for which the proposed EICT based diagnostic tool and monitoring system will be of great help. This system can give legal evidence and for regulatory authorities it will be a practical tool.

The growth of modern technologies in electronics, computers, and communications with availability of hardware for data mining and warehousing technologies, it is easy to realize the diagnostic tool and monitoring system proposed in this paper.

The EMF radiation from cellular mobiles and transmission towers is continuous and additive in nature. Stricter EMF radiation norms are required to be enforced. It is clear that we society has come up with alternate solutions for automobiles air pollution through unleaded petrol, CNG driven vehicles and hybrid vehicles. Similarly there is a need to come up with alternate solutions for EMF radiation problems and mandatory to monitory the EMF levels.

Cellular phone industries are multibillion dollar companies and products are linked to illness. Generally these Industries deny any health problem in spite of large number of health problems reported by many researchers from many countries. Mobile companies should not be in denial mode and have to accept that this is a real world problem.

Monitoring proposed in this research paper is the first step by regulatory authorities given the reason that in various countries the exposures limits vary from $12 \mathrm{w} / \mathrm{m}^{2}$ to $0.00001 \mathrm{w} / \mathrm{m}^{2}$. Entire world has to come up and should have a single exposure limit which is sufficient for cellular mobiles operation for sustainable environmental safety.

\section{REFERENCES}

[1] Balmori Martinez, A. (2003) The effects of microwaves on the trees and the other plants. Valadolid, Spain,

[2] Balmori, A (March 2009) Electromagnetic pollution from phone masts. Effects on wildlife, Pathophysiology. 2009 March 3.

[3] Balmori, A. \& Hallberg, O. (2007) The urban decline of the house sparrow (Passer Domesticus): a possible link with electromagnetic radiation. Electromagnetic biology and medicine, 26,141-151.

[4] Sainudeen Sahib. (2011) impact of mobile phones on the density of Honey Bees. Munis Entomology and Zoology, 6, 396-399.

[5] http://www.next.up.org/pdf/Brazil, New study direct link to 4924 cancer deaths from cellular antennas radiation 28072011 pdf.

[6] Wolf R et al, International Journal of Cancer Prevention, 1(2) April 2004.

[7] Eger H et al, Umwelt Medizin Gesellschaft, 17, 4, 2004.

[8] Berlin, Head of Cancer Registry, 2006.

[9] Santini R et al, Pathol Biol (Paris) 2002 July; 50(6) : 369-373.

[10] Report on possible Impacts of communication Towers on wildlife including Birds and Bees, Ministry of Environment and Forest, Govt of India, 30 Aug 2011.

[11] ICNIRP International Exposure Standards and Guidelines, http://www.icnirp.de/documents/emfgdl.pdf.

[12] Haumann Thomas, et al, HF Radiation levels of GSM cellular phone towers in residential areas, Germany.

[13] http://thepeopleschronicle.in

\section{AUTHOR's INFORMATION}

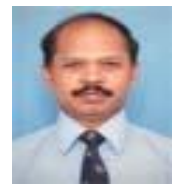

K Parandham Gowd obtained B.Tech in Electronics and Communication Engineering with distinction from S.V. University, Tirupati. In 1994 he has conducted RCS Reduction experiments on coated (by pasting of absorber sheets) and uncoated scaled models of aircraft during his ME (Microwaves and Radar)as thesis work which is first time in India at IIT Roorkee. He has 48 research publications and 06 Technical reports to his credit most of them on RCS/RCS Reduction/Stealth Technology. He has one copyright to his credit on Dynamic RCS Range Validation Procedure from Govt of India. He is a Life Member of All India Management Association (AIMA), AeSI and Fellow of IETE. He had authored a book on Stealth Aircraft Technology in Hindi and English.

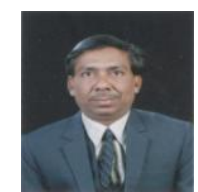

Dr R P Gupta is PhD, ME \&MBA. He has 37 years of experience in Industrial Research \& Development; Production; Quality Management;ISO Certification in Indian telephone Industries and Optel Communications Ltd;Bhopal. Since 2003 he is associated with Engineering colleges in the capacity of Principal/Director in BITS, Globus Engg College, RGPM, Surabhi college of Engineering and Scope College Bhopal. Presently he is life member of nine societies. His area of interests are Satellite communication, Nanotechnology and book writing. Presently he is Chairman IETE Centre (MP \&CG), Bhopal and Director Development at Radharaman Group of Institutions, Bhopal. 